\title{
Exploiting probability density function of deep convolutional autoencoders' latent space for reliable COVID-19 detection on CT scans
}

\author{
Sima Sarv Ahrabi ${ }^{1}$ - Lorenzo Piazzo ${ }^{1}$ - Alireza Momenzadeh ${ }^{1}$. \\ Michele Scarpiniti ${ }^{1}$ (D) Enzo Baccarelli ${ }^{1}$
}

Accepted: 30 January 2022 / Published online: 24 February 2022

(c) The Author(s) 2022

\begin{abstract}
We present a probabilistic method for classifying chest computed tomography (CT) scans into COVID-19 and non-COVID-19. To this end, we design and train, in an unsupervised manner, a deep convolutional autoencoder (DCAE) on a selected training data set, which is composed only of COVID-19 CT scans. Once the model is trained, the encoder can generate the compact hidden representation (the hidden feature vectors) of the training data set. Afterwards, we exploit the obtained hidden representation to build up the target probability density function (PDF) of the training data set by means of kernel density estimation (KDE). Subsequently, in the test phase, we feed a test CT into the trained encoder to produce the corresponding hidden feature vector, and then, we utilise the target PDF to compute the corresponding PDF value of the test image. Finally, this obtained value is compared to a threshold to assign the COVID-19 label or non-COVID-19 to the test image. We numerically check our approach's performance (i.e. test accuracy and training times) by comparing it with those of some state-of-the-art methods.
\end{abstract}

Keywords Deep convolutional autoEencoder - Kernel density estimation · Reconstruction error · Hidden representation · COVID-19

Michele Scarpiniti

michele.scarpiniti@uniroma1.it

Sima Sarv Ahrabi

sima.sarvahrabi@uniroma1.it

Lorenzo Piazzo

lorenzo.piazzo@uniroma1.it

Alireza Momenzadeh

alireza.momenzadeh@uniroma1.it

Enzo Baccarelli

enzo.baccarelli@uniroma1.it

1 Department of Information Engineering, Electronics and Telecommunications, Sapienza University of Rome, Via Eudossiana 18, 00184 Roma, Italy 


\section{Introduction}

There is general agreement on chest computed tomography (CT) scans having a potential role in diagnosing COVID-19 and being particularly effective when used as a complement to polymerase chain reaction (PCR) testing [1-5]. Clearly, cheaper testing methods exist, like lateral flow test. Moreover, the radiation dose inflicted to the patient by a scan may be harmful. However, the method can be useful when a different test is unavailable or too expensive or when a quick diagnosis is needed.

Due to its noteworthy detection capabilities, deep learning (DL) is often employed to assist the evaluation of CT scans for the diagnosis of COVID-19. Indeed, classification of chest CT scans is an important and active research area. In recent contributions, classification is carried out by exploiting supervised DL, using only a few classes of chest CTs (for instance, COVID-19 class and normal one). However, the symptoms of chest diseases manifest themselves in a broad spectrum of visual characteristics. Hence, supervised-trained models could run into trouble when they are fed with chest CTs that do not belong to any of the classes used in the training phase. This issue can be addressed by taking advantage of unsupervised DL, where the neural network models are trained only on data sets of the COVID-19 class. In this manner, the trained model can differentiate the COVID-19 class (the target class) from any other types of chest images (anomalies).

This paper develops an unsupervised classification approach based on autoencoders (AEs). An AE is composed of an encoder and a decoder [6]. The encoder takes an image and transforms it into a hidden feature vector, a compressed input representation. The decoder is used to reconstruct the input image from the hidden feature vector. Since deep convolutional neural networks (CNNs) have proved their effectiveness in image processing and feature extraction, in this paper, we design a deep convolutional autoencoder (DCAE) as the neural network model [7].

We train the proposed DCAE, in an unsupervised manner, on a data set of chest CT scans obtained from COVID-19 patients. After training, the encoder is used to obtain the hidden feature vectors of the training set CT scans. These hidden feature vectors are, in turn, exploited to estimate the probability density function (PDF) of COVID-19 hidden feature vectors, by means of the multivariate kernel density estimation (KDE) method. The classification of a test image is performed by feeding it into the trained encoder to produce its hidden feature vector. Afterwards, the PDF is used to compute the PDF value of the test image. Finally, the obtained PDF value is compared to a suitably tuned threshold to classify the test image as either COVID-19 or non-COVID-19.

For comparison, we also consider a second method for classification based on the DCAE reconstruction error. The resulting error is noticeably higher than the average error corresponding to the training set instances when the trained DCAE is fed with a CT scan of a non-COVID-19 case and attempts to recover it. If this error is below a suitably tuned threshold, the image is classified as COVID-19, otherwise as non-COVID-19.

The rest of the paper is organised as follows: In Sect. 2, we review the related work and present the contributions of this paper. In Sect. 3, we provide details of 
the used data sets and pre-processing tasks. Sections 4 and 5 describe the proposed DCAE architecture and classification approach, respectively. We discuss the obtained numerical results in Sect. 6 and compare the performance of our approach to the ones of some state-of-the-art approaches that rely on the supervised training method. Finally, in Sect. 7, we point out the summarised observations and provide some hints for future research.

\section{Related work and paper contributions}

The literature on the application of DL-based algorithms to the detection of COVID19 is vast. The small volume of available data on COVID-19 patients has motivated the researchers to take this deficiency into account. For instance, the transfer learning approach is adopted in [8-17] to deal with the lack of large-size data sets. In [18], the authors utilise GoogleNet and ResNet for supervised COVID-19 classification. The authors of [19] take a statistical method to address issues like huge computational complexity and large datasets required by deep networks. The adopted approach is based on the evaluating and comparing the statistical representation of medical images. The authors of [20] consider an unbalanced-data supervised algorithm and obtained good results comparable with benchmark architectures.

A large number of research papers adopt supervised learning approaches [21-31]. In [21], the authors consider a binary classification problem and apply the off-theshelf VGG-16. In [26], the authors use depth-wise convolutions with varying dilation rates to extract more diversified features of chest images. The authors use a pretrained model and reach the overall $90.2 \%$ accuracy. In [28], the authors design a neural network model as a combination of convolutional and capsule layers, called COVID-FACT. Despite their great effort, the considered model achieves the $90.82 \%$ accuracy. The authors of [29] propose a model based on the pre-trained ResNet50 and achieve the accuracy, rather similar to the original ResNet50. The DenseNetbased approach, considered in [31], achieves 92\% accuracy. The work by [32] compares different classification architectures on a specific dataset to discover the most suitable real-world scenarios.

The approaches developed in all these papers typically deal with only a limited class of chest images. Hence, a naturally posed question is: to which category does a CT fit when it does not belong to any of the classes learned by the supervisedtrained models? In any case, the aforementioned supervised methods must be trained on both COVID-19 and non-COVID-19 CTs.

Lastly, we provide an overview of AE-based approaches. The authors of [33] consider a stacked AE (SAE), composed of four CAEs, followed by a dense layer, and a final softmax classifier. Each layer is equipped with regularisation to improve the local optimum. The binary classification task, considered by the authors, occurs at the last stage, where the softmax classifier obtains the probability of the two types of labels and performs the classification task with an average accuracy of $94.7 \%$. This method is different from our approach because we perform the classification by directly using the PDF of the hidden feature vectors, without inserting any classifier at the top of our model. The deep convolutional denoising AE, proposed in [34], is 
trained on COVID-19, pneumonia, and a few other types of chest X-rays. Then, the hidden feature vector of a test image is compared to the features of the selected training data sets. The considered AE exhibits good performance. However, unlike our work, this approach relies on training the considered model over each selected class and therefore cannot detect chest CTs except those that belong to the classes of training data sets. The work by [35] focuses on a two-stage learning method and a triple classification task. The authors train their considered model on classes of COVID19, pneumonia, and normal cases separately. Once the hidden feature vectors of all classes are independently obtained, a feature classifier is employed and trained-in a supervised manner-to detect each decision class. The considered approach reaches a quite good accuracy of $93.50 \%$. In contrast to this work, we train our DCAE model on only one class, i.e. the COVID-19. The paper by [36] is based on a variational autoencoder (VAE) model for COVID-19 classification. The VAE model involved adaptive Wiener filtering (AWF)-based pre-processing technique to enhance the image quality. Besides, Inception v4 with Adagrad technique is employed as a feature extractor and unsupervised VAE model is applied for the classification process. As the last research paper, the method introduced by [37] builds a robust statistical generating target histogram of the deep denoising convolutional autoencoder's (DDCAE) latent vector. It then estimates the statistical distance between unknown and target histograms to classify the images according to proper thresholds.

A brief overview of the given literature is provided in Table 1. Finally, the main contributions of this paper are listed below:

- we base the classification task on exploiting an unsupervised deep neural network model, which is trained only on COVID-19 images and, in so doing, we strengthen the robustness of the proposed model concerning the presence of CT scans of other diseases which are not seen during training phase;

- we propose an ad hoc DCAE with an optimised number of layers for the best classifying test performance;

- as an additional novelty, we base the classification task on the estimation of the probability density of the training hidden feature vectors by adopting the KDE method; and finally,

- we carry out numerical tests under benchmark data sets available in the literature and compare the performance of our approach to the ones of some supervised/ unsupervised deep neural networks, both in terms of test accuracy and processing times.

\section{Utilised data sets and pre-processing}

The training data set used in this paper is composed of 4000 CTs of COVID-19 cases collected from over 500 patients. These CT scans have been selected from the 'COVIDx CT-2A' data set; the 'A' variant of the '2nd' version of the open-source 'COVIDx-CT' data set [38]. The 'COVIDx CT-2A' data has been validated by medically qualified personnel. The data are split into training and validation sets containing $80 \%$ and $20 \%$ of instances, respectively. 
Table 1 A synoptic overview of main related work on COVID-19 detection/classification

\begin{tabular}{|c|c|c|}
\hline Work & Algorithm specifications & Accuracy \\
\hline Ref. [8] & $\begin{array}{l}\text { - Modified inception } \\
\text { - BC (Pneumonia, COVID) } \\
\text { - Transfer learning }\end{array}$ & 0.8502 \\
\hline Ref. [10] & $\begin{array}{l}\text { - ResNet50V2/Xception + Softmax } \\
\text { - BC (Normal, COVID) } \\
\text { - Transfer learning }\end{array}$ & 0.9849 \\
\hline Ref. [13] & $\begin{array}{l}\text { - AlexNet/GoogleNet/ResNet + Softmax } \\
\text { - MC (Normal, COVID, Tumour) } \\
\text { - Transfer learning }\end{array}$ & 0.9905 \\
\hline Ref. [16] & $\begin{array}{l}\text { - Modified AlexNet } \\
\text { - BC (Normal, COVID) }\end{array}$ & 0.9410 \\
\hline & - Transfer learning & 0.9410 \\
\hline Ref. [17] & $\begin{array}{l}\text { - DensNet-121 } \\
\text { - MC (Normal, COVID, Cancer) } \\
\text { - Transfer learning }\end{array}$ & 0.8711 \\
\hline Ref. [18] & $\begin{array}{l}\text { - AlexNet/GoogleNet/VGG-16 } \\
\text { - MC (COVID, Atypical/Viral Pneumonia) } \\
\text { - Transfer learning }\end{array}$ & 0.9951 \\
\hline Ref. [20] & $\begin{array}{l}\text { - Deep CNN } \\
\cdot \text { MC (Normal, COVID, Bacterial Pneumonia) }\end{array}$ & 0.9943 \\
\hline Ref. [21] & $\begin{array}{l}\cdot \text { SRGAN/VGG-16 } \\
\cdot \text { BC (Normal, COVID) }\end{array}$ & 0.9800 \\
\hline Ref. [25] & $\begin{array}{l}\cdot \text { Deep CNN + SVM } \\
\cdot \text { MC (Normal, COVID, Bacterial Pneumonia) }\end{array}$ & 0.9866 \\
\hline Ref. [29] & $\begin{array}{l}\text { - ResNet50 + FPN } \\
\text { - MC (Normal, COVID, Bacterial Pneumonia) }\end{array}$ & 0.9300 \\
\hline Ref. [31] & $\begin{array}{l}\text { - DenseNet } \\
\text { - BC (Normal, COVID) }\end{array}$ & 0.9500 \\
\hline Ref. [33] & $\begin{array}{l}\text { - SAE + Softmax Classifier } \\
\cdot \text { BC (Normal + COVID) }\end{array}$ & 0.9470 \\
\hline Ref. [34] & $\begin{array}{l}\text { - Denoising AE + Hidden Features } \\
\text { - MLC (COVID, Pneumonia, and other classes) }\end{array}$ & - \\
\hline Ref. [35] & $\begin{array}{l}\cdot \mathrm{AE}+\mathrm{FPN}+\text { Classifier } \\
\cdot \mathrm{MC} \text { (Normal, COVID, Pneumonia) }\end{array}$ & - \\
\hline Ref. [36] & - $\mathrm{VAE}+\mathrm{AWF}$ & 0.9920 \\
\hline Ref. [37] & $\begin{array}{l}\text { - DDCAE + Latent Space } \\
\text { - (Pneumonia \& Normal) }\end{array}$ & 1.0000 \\
\hline
\end{tabular}

BC, Binary classification; MC, Multiclassification; MLC, Multilabel classification; UC, Unary classification 
Table 2 Train/validation/test set compositions and related web links; WP: Web Page

\begin{tabular}{llllll}
\hline Data set & COVID-19 & Pneumonia & Cancers & Normal & Link \\
\hline Training & 3200 & --- & --- & --- & WP1 $^{1}$ \\
Validation & 800 & --- & --- & --- & WP1 \\
Test & 2500 & 300 & 900 & 300 & WP1 \& WP2 ${ }^{2}$ \\
\hline
\end{tabular}

${ }^{1}$ https://kaggle.com/hgunraj/covidxct

2 https://kaggle.com/mohamedhanyyy/chest-ctscan-images

The test data set comprises 4000 CT slices of COVID-19, normal, pneumonia, and three types of lung cancers, namely adenocarcinoma, large cell carcinoma and squamous cell carcinoma. The test images have been randomly selected from two separate data sets $[38,39]$, composed of CTs of over 500 patients different from those involved in the training set. The data sets include both male and female patients and cover a wide age range.

The information about the employed training/test data sets is summarised in Table 2, where we also give the links to the corresponding web pages. For illustrative purpose, in Fig. 1, we present some instances of various chest CT categories, which are drawn from training and test data sets. As can be seen from the figure, the COVID-19 image is characterised by the presence of several opacities. Indeed, commonly reported imaging features specific of COVID-19 pneumonia are peripheral, bilateral, ground-glass opacities with or without visible intralobular lines: see [40] for a wider discussion.

As a pre-processing task, the margin of every CT is cropped before performing the training phase. Moreover, all the images are resized to 300 pixels in width and 200 pixels in height: this size was selected as a compromise between computational complexity and resolution. Two samples of the COVID-19 dataset in the original, cropped and resized versions are demonstrated in Fig. 2.

\section{The proposed DCAE architecture}

A DCAE is composed of an encoder and a decoder as depicted in Fig. 3. The encoder takes an image as input and transforms it into a hidden feature vector called latent space. The decoder takes the hidden feature vector and attempts to recover the input image. The difference between the input and the output images results in the reconstruction error, which is the cost function to minimise during the training phase. We point out that the hidden feature vectors have fewer dimensions than those of the input images since the DCAE produces compressed versions.

The DCAE, exploited in this work, has been designed to take account of the tradeoff between test classification accuracy and model complexity. Its "ad hoc" designed architecture is detailed in Table 3: the encoder is composed of three convolutional 


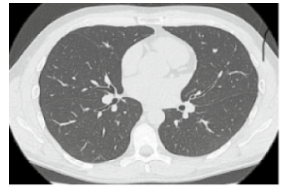

(a) Normal.

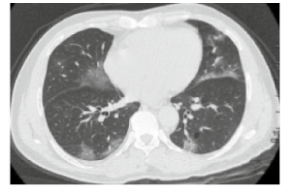

(b) COVID-19.

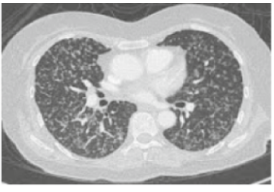

(c) Cancer.

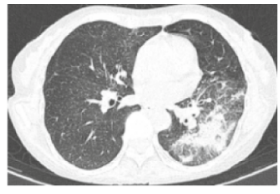

(d) Pneumonia.

Fig. 1 Samples of four lung CT scans drawn from the used training and test data sets

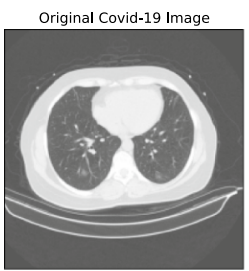

(a) Sample 1
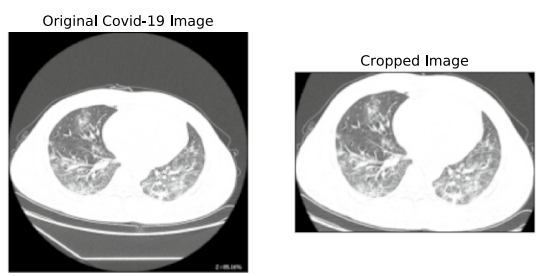

(b) Sample 2

Fig. 2 Two samples of original COVID-19 CTs and their cropped versions

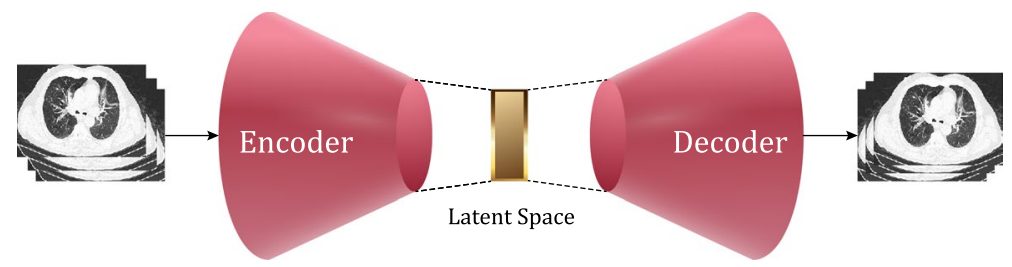

Fig. 3 The autoencoder architecture

layers, two batch normalisation layers, two max-pool layers, followed by flatten and dense layers with rectifier activation functions (ReLUs).

The DCAE is trained to recover its input, i.e. COVID-19 CT scans. As training cost function, we use the mean squared error (MSE), and in order to minimise the considered cost function over the training set, we employ the Adam (Adaptive moment estimation) solver, which is a gradient-based stochastic optimisation algorithm that takes account of the first and second moments of underlying gradients [41]. The training is carried out over 100 epochs. After the training phase, we select the DCAE weights, which give rise to the minimum squared error on the validation data set. Table 4 recap details on the adopted model setting. For illustrative purpose, Fig. 4 presents the graphs of MSE and accuracy versus the number of epochs, over which the training phase is carried out.

Note that we have considered other options for both the architecture and the training parameters, using several alternatives proposed in the literature. The various options have been compared by means of unreported tests: the final architecture and 
Table 3 The architecture of proposed DCAE; encoder input shape: $200 \times 300 \times 3$; decoder input shape: 128 ; number of parameters of the encoder: 31, 097, 408; number of parameters of the decoder: $31,337,475$
Table 4 Main parameters of the carried out training phase

\begin{tabular}{llll}
\hline Layer & Kernel & Stride & Output shape \\
\hline Encoder & & & \\
Conv2D & $3 \times 3$ & - & $200 \times 300 \times 256$ \\
BatchNorm & - & - & $200 \times 300 \times 256$ \\
Conv2D & $3 \times 3$ & - & $200 \times 300 \times 128$ \\
MaxPool2D & $2 \times 2$ & - & $100 \times 150 \times 128$ \\
Conv2D & $3 \times 3$ & - & $100 \times 150 \times 64$ \\
BatchNorm & - & - & $100 \times 150 \times 64$ \\
MaxPool2D & $2 \times 2$ & - & $50 \times 75 \times 64$ \\
Flatten & - & - & 240000 \\
Dense & - & - & 128 \\
Decoder & & - & 240000 \\
Dense & - & - & $50 \times 75 \times 64$ \\
Reshape & $3 \times 3$ & 2 & $100 \times 150 \times 128$ \\
Conv2DTranspose & - & - & $100 \times 150 \times 128$ \\
BatchNorm & $3 \times 3$ & 2 & $200 \times 300 \times 256$ \\
Conv2DTranspose & - & - & $200 \times 300 \times 256$ \\
BatchNorm & $3 \times 3$ & 1 & $200 \times 300 \times 3$ \\
Conv2DTranspose & & & \\
\hline
\end{tabular}

\begin{tabular}{ll}
\hline Description & Value \\
\hline Batch size & 16 \\
Number of epochs & 100 \\
Optimiser & Adam \\
Learning rate & $1 \times 10^{-5}$ \\
Loss function & MSE \\
Size of hidden feature vectors & 128
\end{tabular}

training parameters have been selected because they yielded the highest classification accuracy.

The DCAE is implemented in Python language, using TensorFlow and Keras API. All the numerical trials have been carried out on a PC equipped with an AMD Ryzen $95900 X$ 12-Core $3.7 \mathrm{GHz}$ processor, two GeForce RTX 3070 graphics cards, and 128 GB RAM. 


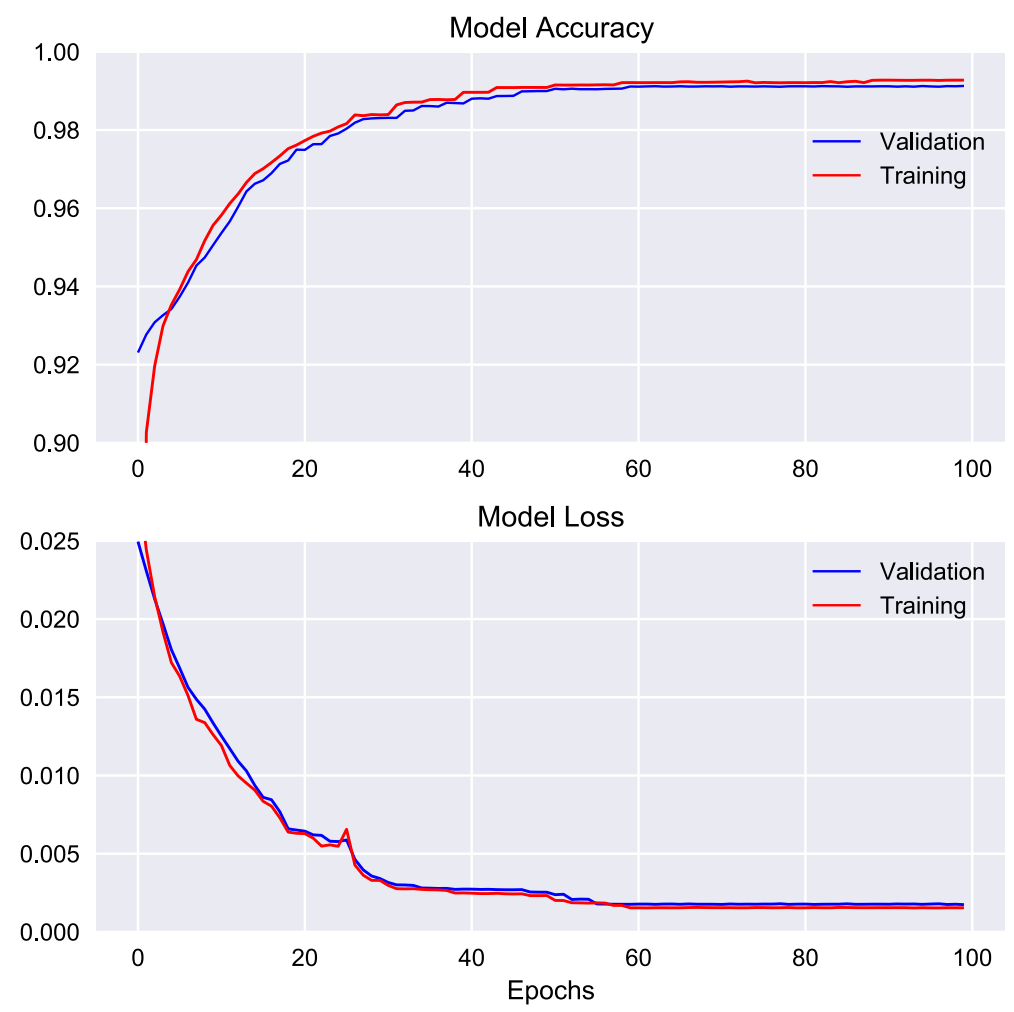

Fig. 4 Numerically evaluated plots of accuracy-vs.-epochs and MSE-vs.-epochs under the training phase

\section{Image classification}

In this section, we describe the classification approach that is based on the PDF estimation of the DCAE hidden feature vectors.

\subsection{PDF estimation of the training hidden feature vectors}

In order to estimate the PDF of the hidden feature vectors, a first choice that needs to be done is whether to use a parametric or a nonparametric method. Since we have no clues on the shape of the pdf and we do not want to polarise the estimation by guessing, we decided to use a nonparametric estimate. Among the nonparametric methods, we selected the KDE approach, ${ }^{1}$ which is well known [42] and outperforms the simpler histogram approach.

\footnotetext{
1 The KDE is a traditional density estimation method. Such methods may become unmanageable when the number of variables is too high. Luckily, the computational power of modern computers makes this method suitable for the problem at hand.
} 


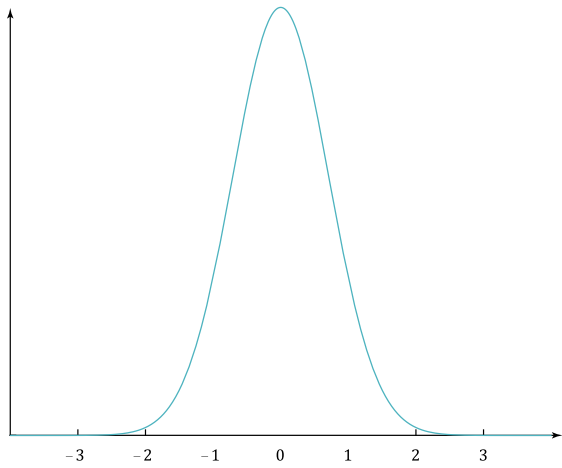

(a) The Gaussian kernel.

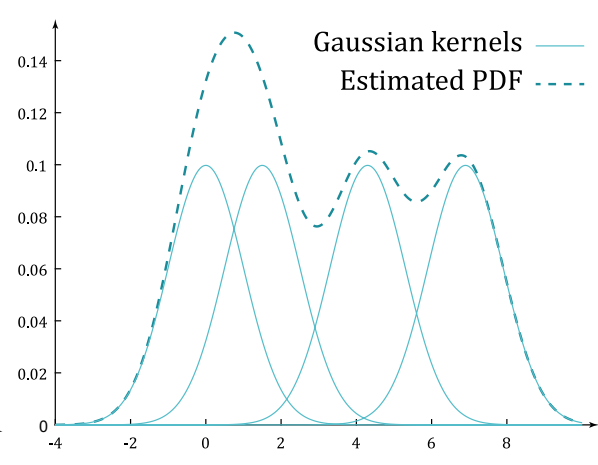

(b) Example of the kernel-based estimation.

Fig. 5 An example of KDE estimation over $n=4$ data points: a copy of the kernel is placed on each data point and the copies are summed to produce the final PDF estimate

The KDE method is based on a univariate kernel function denoted by $K(x)$. Although a wide variety of different kernels can be used, according to [6], we consider the Gaussian one, i.e. $K(x)=e^{-x^{2}}$ (see Fig. 5a). The kernel is used as an interpolating function to build up the PDF estimate.

In order to describe the KDE approach, we first illustrate it for the simple case of a univariate PDF. Let us consider a set of $n$ real numbers: $x_{i}$ for $i=1, \ldots, n$, drawn from a (hidden) random variable (RV) $X$, which exhibits an unknown PDF, $f_{X}(x)$, that we want to estimate. The KDE estimate of the PDF is [6]:

$$
\bar{f}_{X}(x)=\frac{1}{\alpha} \sum_{i=1}^{n} K\left(\frac{x-x_{i}}{h}\right)
$$

where the constant $\alpha$ is a normalisation factor, used to set the integral of $\bar{f}_{X}(x)$ to one, while the parameter $h$ is the kernel bandwidth which is used to set the width of the kernel. The estimation is illustrated in Fig. 5b, where we see that the density is obtained as the superposition of $n$ scaled and shifted copies of the kernel.

To describe the multivariate case, let us assume that we have a $p$ dimensional RV $X$, with a multivariate density $f_{X}(\mathbf{x})$ where $\mathbf{x} \in \mathbb{R}^{p}$ is a $p$-dimensional vector. Moreover, we have a set of vectors $\mathbf{x}_{i} \in \mathbb{R}^{p}$ for $i=1, \ldots, n$ which are samples drawn from the RV $X$. The KDE estimate generalises to the multivariate case as in:

$$
\bar{f}_{X}(\mathbf{x})=\frac{1}{\alpha} \sum_{i=1}^{n} K\left(\frac{\left\|\mathbf{x}-\mathbf{x}_{i}\right\|}{h}\right),
$$

where II.II denotes the Euclidean norm.

In order to estimate the PDF of the DCAE hidden feature vector when the input is a COVID-19 image, we use the KDE estimate of (2), where the vectors $\mathbf{x}_{i}$ are the hidden feature vectors obtained from the images of the training set, with $n=3200$ and $p=128$ in our setting. For the computation of the KDE estimate, we used 
Scikit-learn [43], an open-source machine learning library developed for the Python environment. One parameter that needs to be carefully tuned is the bandwidth $h$. Indeed, the choice of bandwidth controls the bias-variance trade-off in the estimation of the PDF [6]. This means that a too narrow bandwidth leads to a high-variance estimate (over-fitting), while a too wide bandwidth results in a high-bias estimate (under-fitting). For selecting the bandwidth, we employed the Scikit-learn built-in method of grid search cross-validation. This algorithm selects the best option from a grid of parameter values provided by the user, automating the 'trial-and-error' method. A second option could be to use the maximum likelihood cross-validation (MLCV) approach, introduced in [44, 45].

We point out that the computational complexity of the evaluated KDE-based estimation approach depends on the length $p$ of the hidden feature vectors and the number $n$, of employed training images. specifically, since $n$ Euclidean distances among $p$-dimensional vectors must be computed, the resulting computational complexity scales as:

$$
\mathcal{O}(n \times p) .
$$

\subsection{Classification based on the estimated PDF}

In our approach, the estimated PDF of the DCAE latent space is used to classify the test images. To this end, we feed the image to the DCAE encoder to produce the corresponding hidden feature vector. Next, the obtained vector is used as the argument of the estimated PDF, in order to compute its corresponding PDF value. In practice, since the obtained values of the PDF are minimal, it is more robust to work with $\log$ probability densities. If the obtained value of $\log$ density is above a (suitably tuned) threshold, the image is classified as a COVID-19 case; otherwise, it is labelled as a non-COVID-19 one.

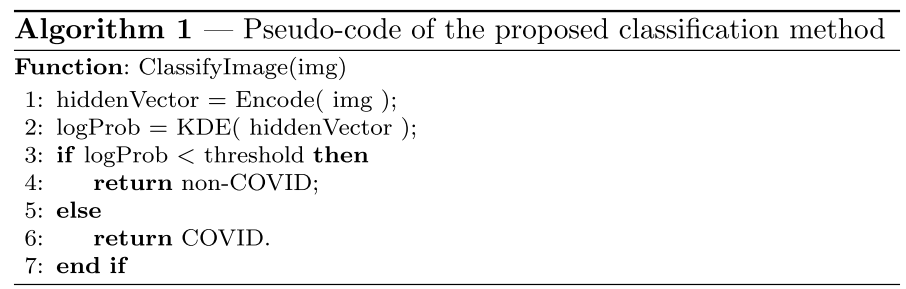

In order to suitably set the decision threshold, we evaluate the real-valued log probability densities of $n$ images in the training set, denoted by $l_{i}$, for $i=1,2, \cdots, n$. Then, we compute the mean and the standard deviation of the evaluated $l_{i} \mathrm{~s}$, denoted by $\mu_{l}$ and $\sigma_{l}$, respectively. Thus, the threshold is set to:

$$
T H=\mu_{l}+\beta \sigma_{l},
$$




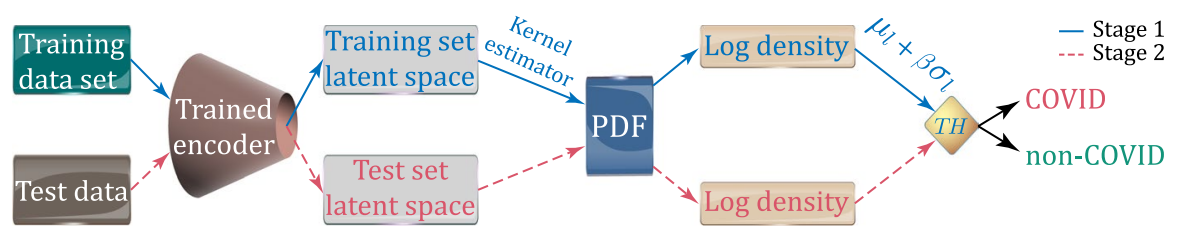

Fig. 6 Estimation of the PDF of the latent space (stage 1) and classification of the test images (stage 2)

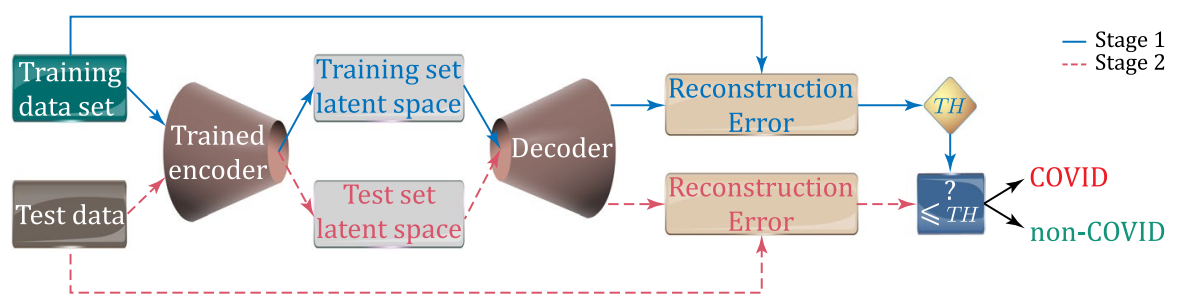

Fig. 7 Evaluation of reconstruction errors of training set and the threshold (stage 1), and classification of the test images (stage 2)

where the constant $\beta$ is evaluated by using the validation set. In particular, we select $\beta$ so that the threshold equals the minimum log probability of the validation set, namely -233.5: in this way the whole validation set is classified as COVID-19. The overall proposed classification procedure is summarised in Fig. 6 and Algorithm 1.

\subsection{A benchmark classifier}

For comparison purposes, we consider a benchmark classification method. To this end, we recall that the DCAE is trained to produce an output similar to the input as much as possible. The difference between the input and the output is the reconstruction error, which minimises the cost function during the training phase. However, since the training set contains COVID-19 scans only, the DCAE is effective at recovering COVID-19 images, but it is ineffective for recovering non-COVID-19 images. Therefore, a high reconstruction MSE can be an index that the image does not belong to the COVID-19 class.

By considering this fact, we build up the following benchmark classification procedure. Given an image, we feed it into the DCAE and compute the Euclidean norm of its reconstruction error. If the obtained error norm is below a (suitably) set threshold, the image is classified as COVID-19. The threshold is evaluated by using the instances of validation set. In particular, we select the threshold equal to the maximum error of the validation set, namely 0.08 : in this way the whole validation set is classified as COVID-19. Figure 7 presents the flow diagram of the classification process based on reconstruction error. 
Table 5 Basic metrics

\begin{tabular}{ll}
\hline Name & Description \\
\hline True positive (TP) & COVID-19 image classified as COVID-19 \\
True negative (TN) & Non-COVID-19 image classified as non-COVID-19 \\
False positive (FP) & Non-COVID-19 image classified as COVID-19 \\
False negative (FN) & COVID-19 image classified as non-COVID-19 \\
\hline
\end{tabular}

Table 6 Utilised performance indexes

\begin{tabular}{ll}
\hline Metrics & Formula \\
\hline Recall & $T P /(T P+F N)$ \\
Precision & $T P /(T P+F P)$ \\
F-score & $2 T P /(2 T P+F P+F N)$ \\
Accuracy & $(T P+T N) /(T P+F N+F P+T N)$ \\
\hline
\end{tabular}

\section{Numerical results and performance comparisons}

In order to evaluate the performance of our classification method, we carried out several tests. We split the presentation into three parts that are: (i) the results that are obtained from the KDE probabilistic approach; (ii) the results that are achieved through reconstruction error evaluation; and (iii) performance comparisons with some state-of-the-art solutions. In the next subsection, we describe the employed performance metrics as a preliminary step.

\subsection{Performance metrics}

Given a binary classifier, the considered performance metrics are the rates of true-positive (TP), true-negative (TN), false-positive (FP) and false-negative (FN) assignments. These metrics are summarised in Table 5. These basic metrics can be represented in a compact form as the four elements of the resulting confusion matrix [46].

From these basic metrics, a number of affiliated performance indexes can be derived [46]. This paper will consider accuracy, recall, precision and F1-score, as performance indexes. The formal definitions of these indexes are given in Table 6 .

\subsection{Performance of the proposed approach}

As a first experiment, we carried out a classification of the test data set by using the proposed approach. To this end, each test image is fed to the trained DCAE, and the corresponding hidden feature vector is obtained. Afterwards, the obtained 


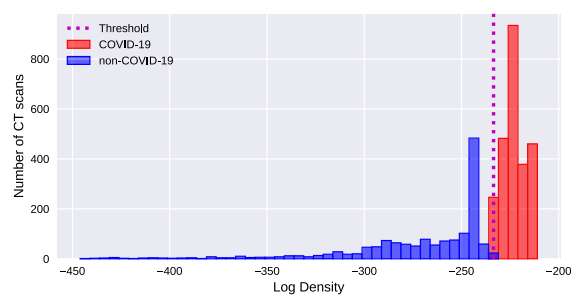

(a) Distribution of log densities on 4000 test CTs.

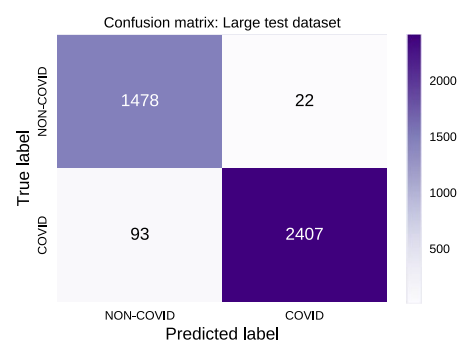

(b) Corresponding confusion matrix.

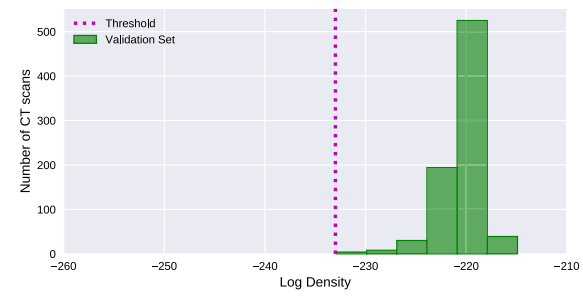

(c) Distribution of log densities on 800 CTs of validation set.

Fig. 8 Results under the proposed approach

hidden feature vector is used as an argument of the multivariate PDF estimated by the KDE and then the corresponding log density value is computed. The soobtained log densities are plotted in Fig. 8a for all the test images and in Fig. 8c for validation set.

From Fig. 8, we see that the log densities of the COVID-19 images are almost separated from those of the non-COVID-19 ones. In Fig. 8a and c, the vertical dashed line denotes the threshold, which is laid between the two classes. As a result, the proposed method achieves a $97.12 \%$ test accuracy. The corresponding confusion matrix is presented in Fig. 8b. The last row of Table 7 reports the evaluation performance metrics.

\subsection{Performance of the benchmark classifier}

As a second experiment, we evaluate the performance of the benchmark classification approach of Sect. 5.3, which is based on the reconstruction error. To this end, in Fig. 9a, we plot the reconstruction errors obtained by feeding the whole test set to the DCAE, using two different colours for the COVID-19 and non-COVID-19 scans. From the figure, we see that, as expected, the error is lower for the COVID19 images. However, the two classes are not disjoint. The threshold is shown in Fig. 9a by the vertical dashed line. The resulting accuracy is equal to $86.35 \%$. The corresponding confusion matrix is presented in Fig. 9b, and the related performance indexes are reported in Table 7. From Table 7, we conclude that the obtained 


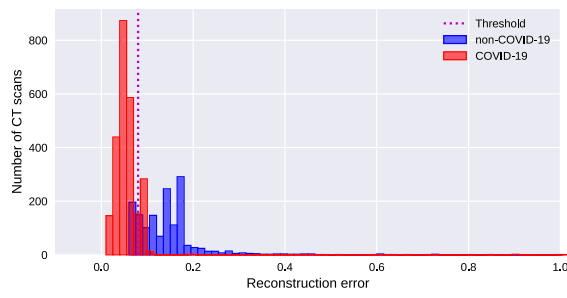

(a) Distribution of reconstruction error over 4000 test CTs.

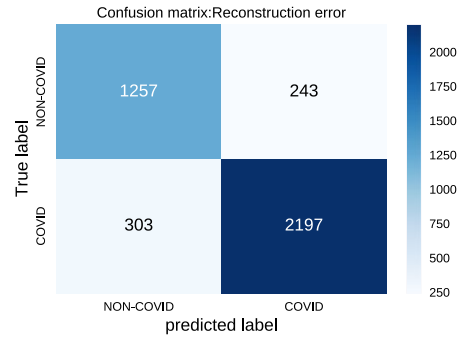

(b) Corresponding confusion matrix

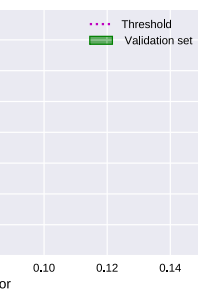

(c) Distribution of reconstruction error on $800 \mathrm{CTs}$ of validation set.

Fig. 9 Performance results of the reconstruction error approach

Table 7 Performance metrics of the proposed approach and the benchmark one based on the reconstruction error

\begin{tabular}{llllll}
\hline Method & Accuracy & Precision & Recall & F1-score & Test CTs \\
\hline Reconstruction error & 0.8635 & 0.8584 & 0.8531 & 0.8555 & 4000 \\
Our approach & 0.9712 & 0.9741 & 0.9659 & 0.9696 & 4000 \\
\hline
\end{tabular}

performance of the classification approach based on the reconstruction error is about $11 \%$ inferior performance than the corresponding one of the KDE-based proposed approach.

\subsection{Performance comparison against state-of-the-art approaches and robustness test}

In this subsection, we study the classification accuracy of several other approaches and compare their results with ours. For computational complexity reasons, we do not use the whole test set but a subset of it, comprising 1000 images (500 COVID, 120 pneumonia, 120 normal and 260 cancer). On this reduced set, our approach reaches a 100\% accuracy, while the reconstruction error approach reaches $97 \%$ accuracy (see Table 8). 
In order to compare our results with those obtained by supervised learning, we have considered three state-of-the-art supervised models, namely GoogLeNet [47], AlexNet [48], and ResNet18 [49], which are typically used for image classification and able to successfully classify out-of-sample examples.

As a first experiment, we have trained - in a supervised way-the aforementioned models, using a training set composed of 2000 COVID-19 CTs and 2000 nonCOVID-19 CTs. The non-COVID-19 set is composed of five classes, namely normal CTs, pneumonia and three types of lung cancers CTs. Afterwards, each model has been evaluated on the reduced test set. The obtained performance indexes are

Table 8 Comparison with supervised classification

Table 9 Robustness tests: pneumonia CTs are present in the test set, but not in the training set for the AlexNet, GoogleNet and ResNet18

\begin{tabular}{lcccc}
\hline Model & Accuracy & Precision & Recall & F1-score \\
\hline AlexNet & 0.9840 & 0.9840 & 0.9841 & 0.9840 \\
GoogleNet & 0.9960 & 0.9960 & 0.9960 & 0.9960 \\
ResNet18 & 0.9930 & 0.9931 & 0.9930 & 0.9930 \\
Unsupervised approach & & & & \\
Reconstruction error & 0.9710 & 0.9710 & 0.9710 & 0.9710 \\
Our approach & 1.0000 & 1.0000 & 1.0000 & 1.0000 \\
\hline
\end{tabular}

The training set is composed of 4000 images (COVID only for the proposed method and belonging to five different classes for the supervised methods). The test set is composed of 1000 images (500 COVID, 120 pneumonia, 120 normal and 260 cancer)

\begin{tabular}{lcccc}
\hline Model & Accuracy & Precision & Recall & F1-score \\
\hline AlexNet & 0.9060 & 0.9060 & 0.9209 & 0.9052 \\
GoogleNet & 0.9140 & 0.9140 & 0.9266 & 0.9134 \\
ResNet18 & 0.9130 & 0.9130 & 0.9259 & 0.9123 \\
Unsupervised approach & & & \\
Reconstructio error & 0.9710 & 0.9710 & 0.9710 & 0.9710 \\
Our approach & 1.0000 & 1.0000 & 1.0000 & 1.0000 \\
\hline
\end{tabular}

The test set is composed of 1000 images (500 COVID, 120 pneumonia, 120 normal and 260 cancer)

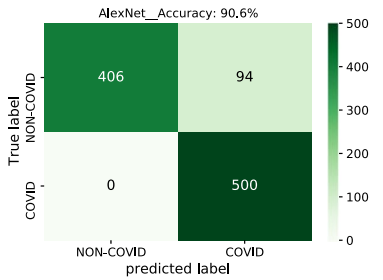

(a) AlexNet

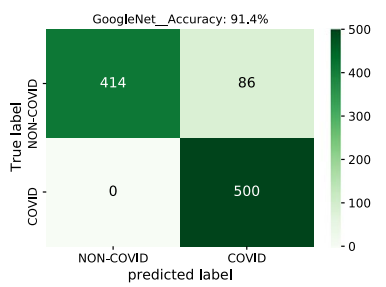

(b) GoogleNet

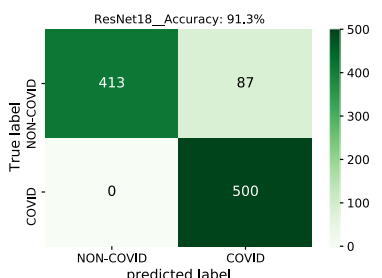

(c) ResNet18

Fig. 10 Confusion matrices: pneumonia CTs are present in test data set, but not in the train data set 
Table 10 Comparative analysis of binary classification of COVID-19 vs non-COVID-19 based on similar datasets
Table 11 Average test times over a batch of 10 images

\begin{tabular}{lllll}
\hline Model & Accuracy & Precision & Recall & F1-score \\
\hline HB-DDCAE & 1.0000 & 1.0000 & 1.0000 & 1.0000 \\
SVM-2D & 0.9340 & 0.9417 & 0.9340 & 0.9378 \\
SVM-3D & 0.9270 & 0.9363 & 0.9270 & 0.9316 \\
SVM-RBF & 0.9170 & 0.9788 & 0.9170 & 0.9469 \\
MLP-50 & 0.7600 & 0.8132 & 0.7600 & 0.7857 \\
MLP-100 & 0.7740 & 0.7874 & 0.7740 & 0.7806 \\
MLP-200 & 0.7830 & 0.8123 & 0.7830 & 0.7974 \\
RF-100 & 0.7110 & 0.7852 & 0.7110 & 0.7463 \\
RF-500 & 0.7200 & 0.7903 & 0.7200 & 0.7535 \\
RF-1000 & 0.7210 & 0.7893 & 0.7210 & 0.7536 \\
\hline
\end{tabular}

The test set is composed of 1000 images (500 COVID, 500 nonCOVID)

\begin{tabular}{ll}
\hline Model & Test time $(s)$ \\
\hline AlexNet & 1.3566 \\
GoogleNet & 1.2400 \\
ResNet18 & 2.4754 \\
Proposed & 1.0026 \\
\hline
\end{tabular}

reported in Table 8. These results are comparable to those obtained from the KDEbased approach.

While the supervised DL models have a performance similar to our approach, we expect that their performance is more sensitive (i.e. less robust) to unseen test images. To corroborate this statement, we carry out a final experiment, where we retrain all the supervised models using a modified data set: we eliminate pneumonia CTs from the non-COVID-19 images, replace them with the normal CTs, apply all the procedures from scratch. Once the best weights are achieved, we perform the test phase on the reduced test set, including pneumonia CTs. The corresponding performance indexes are presented in Table 9, while the confusion matrices are shown in Fig. 10. It is observed that the supervised models are able to distinguish COVID-19 perfectly, but since the pneumonia images have not been included in the training phase, the models are in trouble with these images. In other words, if some classes of images are not present in the training set, the supervised-trained models are not capable of correctly classifying them in the test phase. We conclude that our approach is more robust in the presence of outliers in the test set.

As a second experiment, we compare our approach with several other methods presented in [37]. In particular, we consider the Histogram-Based DDCAE (HBDDCAE) method proposed in that work, together with several shallow methods. The shallow methods are: support vector machine (SVM) with 2-degree (SVM-2D) and 3-degree (SVM-3D) polynomial, and radial basis function (SVM-RBF) kernels; multilayer perceptron (MLP) equipped with single hidden layers composed by 50 
(MLP-50), 100 (MLP100), and 200 (MLP-200) neurons; random forest (RF) composed by 100 (RF-100), 500 (RF-500), and 1000 (RF-1000) binary trees. See [37] for details about the training. The results are presented in Table 10.

From Table 10, we note that the shallow algorithms have an inferior performance. On the other hand, the HB-DDCAE method has the same performance of the proposed approach.

\subsection{Test-time comparisons}

Table 11 reports the (numerically evaluated) average times required by the implemented methods for classifying a batch of 10 images in the test phase. A comparison of the entries of this table leads to the conclusion that the average test time of our method is over $25 \%$ less than the corresponding ones of the implemented benchmark models. We have numerically ascertained that this is due to the fact that our proposed method works on the reduced-size (i.e. compressed) hidden feature vectors, while all the benchmark models directly process the full-size input test images. This conclusion provides further support about the actual effectiveness of the proposed KDE-based classifying approach.

\section{Conclusion and hints for future research}

We propose a method for classifying lung CTs as COVID-19 or non-COVID-19. The method exploits a DCAE trained on COVID-19 CTs only and a KDE estimation of the PDF of the DCAE hidden feature vectors. The DCAE is used to produce the corresponding hidden feature vector to classify an image. Afterwards, we use the soobtained PDF evaluation to compute, in the test phase, the PDF value of the hidden feature vector, corresponding to a test image: if the PDF value is above a suitable threshold, that image is classified as COVID-19, otherwise as non-COVID-19.

We compare our KDE-based approach to the benchmark method that is based on the reconstruction error. In addition, we also check the accuracy performance of three widely known supervised models and the results of some recent papers. The carried out tests support the conclusion that the proposed approach is highly effective in terms of both achieved test accuracy and the needed test times.

The presented results could be extended, at least, along two main research lines. A first research line could concern the utilisation of generative adversarial networks (GANs) for the generation of additional training examples in the case of new COVID-19 mutations (as, for example, the Omicron one), in order to quickly provide reliable automatic detection of these mutations without pausing for the acquisition of sufficiently large new datasets. A second research line could regard the implementation of the proposed algorithmic framework atop distributed Fog/Cloud networked technological platforms $[50,51]$, in order to be capable to quickly generate reliable clinical diagnosis by leveraging the low-delay and (possibly, adaptive 
[52] and /or multiantenna empowered [53, 54]) capability of emerging Fog computing platforms for allowing ubiquitous wireless access to computing-demanding medical diagnostic services.

Acknowledgements This work has been supported by the projects: "SoFT: Fog of Social IoT" funded by Sapienza University of Rome Bando 2018 and 2019; "End-to-End Learning for 3D Acoustic Scene Analysis (ELeSA)" funded by Sapienza University of Rome Bando Acquisizione di medie e grandi attrezzature scientifiche 2018; and, "DeepFog-Optimized distributed implementation of Deep Learning models over networked multitier Fog platforms for IoT stream applications" funded by Sapienza University of Rome Bando 2020.

\section{Declarations}

Conflict of interest The authors declare no conflict of interest.

Open Access This article is licensed under a Creative Commons Attribution 4.0 International License, which permits use, sharing, adaptation, distribution and reproduction in any medium or format, as long as you give appropriate credit to the original author(s) and the source, provide a link to the Creative Commons licence, and indicate if changes were made. The images or other third party material in this article are included in the article's Creative Commons licence, unless indicated otherwise in a credit line to the material. If material is not included in the article's Creative Commons licence and your intended use is not permitted by statutory regulation or exceeds the permitted use, you will need to obtain permission directly from the copyright holder. To view a copy of this licence, visit http://creativecommons.org/licen ses/by/4.0/.

\section{References}

1. Ariana Axiaq, Ahmad Almohtadi, Samuel A Massias, et al (2021) The role of computed tomography scan in the diagnosis of COVID-19 pneumonia. Current Opinion in Pulmonary Medicine, 27(3):163-168, May https://doi.org/10.1097/MCP.0000000000000765

2. Nayaar Islam, Sanam Ebrahimzadeh, Jean-Paul Salameh et al (2021) Thoracic imaging tests for the diagnosis of COVID-19. Cochrane Database of Systematic Reviews 3(CD013639):1-147. https://doi.org/10.1002/14651858.CD013639.pub4

3. Yicheng Fang, Huangqi Zhang, Jicheng Xie et al (2020) Sensitivity of chest CT for COVID19: comparison to RT-PCR. Radiology 296(2):E115-E117. https://doi.org/10.1148/radiol.20202 00432

4. Tao Ai, Zhenlu Yang, Hongyan Hou et al (2020) Correlation of chest CT and RT-PCR testing for coronavirus disease 2019 (COVID-19) in china: a report of 1014 cases. Radiology 296(2):E32-E40. https://doi.org/10.1148/radiol.2020200642

5. Xingzhi Xie, Zheng Zhong, Wei Zhao et al (2020) Chest CT for typical coronavirus disease 2019 (COVID-19) pneumonia: relationship to negative RT-PCR testing. Radiology 296(2):E41-E45. https://doi.org/10.1148/radiol.2020200343

6. Goodfellow Ian, Bengio Yoshua, Courville Aaron (2016) Deep learning. MIT Press, The MIT Press, November

7. Jonathan Masci, Ueli Meier, Dan Cireşan, et al (2011) Stacked convolutional auto-encoders for hierarchical feature extraction. In Artificial neural networks and machine learning-ICANN 2011, Lecture Notes in Computer Science, vol 6791, pages 52-59, Berlin, Heidelberg, June. Springer. https:// doi.org/10.1007/978-3-642-21735-7_7

8. Shuai Wang, Bo Kang, Jinlu Ma et al (2021) A deep learning algorithm using CT images to screen for Corona Virus disease (COVID-19). European radiology 31:1-9. https://doi.org/10.1007/ s00330-021-07715-1

9. Muhammad Attique Khan, Nazar Hussain, Abdul Majid, et al (2021) Classification of positive COVID-19 CT scans using deep learning. Computers, Materials and Continua, 66(3):2923-2938, January. https://doi.org/10.32604/cmc.2021.013191 
10. Mohammad Rahimzadeh, Abolfazl Attar, Seyed Mohammad Sakhaei (2021) A fully automated deep learning-based network for detecting COVID-19 from a new and large lung CT scan dataset. Biomedical Signal Processing and Control, 68(102588), July. j.bspc.2021.10258

11. Ziwei Zhu, Zhang Xingming, Guihua Tao et al (2021) Classification of COVID-19 by compressed chest CT image through deep learning on a large patients cohort. Interdisciplinary Sciences: Computational Life Sciences 13(1):73-82. https://doi.org/10.1007/s12539-020-00408-1

12. Md Zahangir Alom, M M Shaifur Rahman, Mst Shamima Nasrin, Tarek M. Taha, and Vijayan K. Asari (2020) Covid-mtnet: Covid-19 detection with multi-task deep learning approaches, arXiv platform

13. Tao Zhou, Huiling Lu, Zaoli Yang, et al (2021) The ensemble deep learning model for novel COVID-19 on CT images. Applied Soft Computing, 98(January), January. https://doi.org/10.1016/j. asoc.2020.106885

14. Arshia Rehman, Saeeda Naz, Ahmed Khan, et al (2020) Improving coronavirus (covid-19) diagnosis using deep transfer learning. medRxiv, https://doi.org/10.1101/2020.04.11.20054643

15. Emtiaz Hussain, Mahmudul Hasan, Md Anisur Rahman, et al (2021) Corodet: a deep learning based classification for COVID-19 detection using chest X-ray images. Chaos, Solitons \& Fractals, 142(110495):1-12, January. j.chaos.2020.110495

16. Halgurd S Maghdid, Aras T Asaad, Kayhan Zrar Ghafoor, et al (2021) Diagnosing COVID-19 pneumonia from X-ray and CT images using deep learning and transfer learning algorithms. In Multimodal image exploitation and learning 2021, volume 11734 of SPIE, pages 99-110, Florida, United States, April. SPIE. https://doi.org/10.1117/12.2588672

17. Chun Li, Yunyun Yang, Hui Liang, Boying Wu (2021) Transfer learning for establishment of recognition of COVID-19 on CT imaging using small-sized training datasets. Knowledge-Based Systems 218(106849):1-9. https://doi.org/10.1016/j.knosys.2021.106849

18. Ali Abbasian Ardakani, Alireza Rajabzade Kanafi, U Rajendra Acharya, , et al. Application of deep learning technique to manage COVID-19 in routine clinical practice using CT images: results of 10 convolutional neural networks. Computers in Biology and Medicine, 121(103795):1-9, June 2020. https://doi.org/10.1016/j.compbiomed.2020.103795

19. Michele Scarpiniti, Sima Sarv Ahrabi, Enzo Baccarelli, et al (2021) A histogram-based low-complexity approach for the effective detection of covid-19 disease from CT and X-ray images. Applied Sciences, 11(19):8867, September . https://doi.org/10.3390/app11198867

20. Kai Hu, Yingjie Huang, Wei Huang et al (2021) Deep supervised learning using self-adaptive auxiliary loss for COVID-19 diagnosis from imbalanced CT images. Neurocomputing 458:232-245. https://doi.org/10.1016/j.neucom.2021.06.012

21. Wenjun Tan, Pan Liu, Xiaoshuo Li et al (2021) Classification of COVID-19 pneumonia from chest CT images based on reconstructed super-resolution images and VGG neural network. Health Information Science and Systems 9(1):1-12. https://doi.org/10.1007/s13755-021-00140-0

22. Linda Wang, Zhong Qiu Lin, Alexander Wong (2020) COVID-Net: a tailored deep convolutional neural network design for detection of COVID-19 cases from chest X-ray images. Scientific Reports, 10(1):1-12, November. https://doi.org/10.1038/s41598-020-76550-Z

23. Umut Özkaya, Şaban Öztürk, Mucahid Barstugan (2020) Coronavirus (COVID-19) Classification Using Deep Features Fusion and Ranking Technique, pages 281-295. Springer International Publishing, Cham, https://doi.org/10.1007/978-3-030-55258-9_17

24. Cheng Jin, Weixiang Chen, Yukun Cao, et al (2020) Development and evaluation of an ai system for covid-19 diagnosis. medRxiv, https://doi.org/10.1101/2020.03.20.20039834

25. Prabira Kumar Sethy, Santi Kumari Behera, Pradyumna Kumar Ratha, Preesat Biswas (2020) Detection of coronavirus disease (COVID-19) based on deep features and support vector machine. International Journal of Mathematical, Engineering and Management Sciences, 5(4):643-651, January. IJMEMS.2020.5.4.052

26. Tanvir Mahmud, Md Awsafur Rahman, Shaikh Anowarul Fattah (2020) CovXNet: a multi-dilation convolutional neural network for automatic COVID-19 and other pneumonia detection from chest $\mathrm{X}$-ray images with transferable multi-receptive feature optimization. Computers in biology and medicine, 122(103869), July. https://doi.org/10.1016/j.compbiomed.2020.103869

27. Sima Sarv Ahrabi, Michele Scarpiniti, Enzo Baccarelli, et al (2021) An accuracy vs. complexity comparison of deep learning architectures for the detection of COVID-19 disease. Computation, 9(1), January. https://doi.org/10.3390/computation9010003 
28. Shahin Heidarian, Parnian Afshar, Nastaran Enshaei, et al (2021) COVID-FACT: a fully-automated capsule network-based framework for identification of COVID-19 cases from chest CT scans. Frontiers in Artificial Intelligence, 4(598932), May. https://doi.org/10.3389/frai.2021.598932

29. Ying Song, Shuangjia Zheng, Liang Li et al (2021) Deep learning enables accurate diagnosis of novel coronavirus (COVID-19) with CT images. IEEE/ACM Transactions on Computational Biology and Bioinformatics. https://doi.org/10.1109/TCBB.2021.3065361

30. Suat Toraman, Talha Burak Alakus, Ibrahim Turkoglu (2020) Convolutional capsnet: a novel artificial neural network approach to detect COVID-19 disease from X-ray images using capsule networks. Chaos, Solitons \& Fractals, 140(110122), November. https://doi.org/10.1016/j.chaos.2020. 110122

31. Shuyi Yang, Longquan Jiang, Zhuoqun Cao, et al (2020) Deep learning for detecting corona virus disease 2019 (COVID-19) on high-resolution computed tomography: a pilot study. Annals of Translational Medicine, 8(7):450, March. https://doi.org/10.21037/atm.2020.03.132

32. Andrea Loddo, Fabio Pili, Cecilia Di Ruberto (2021) Deep learning for covid-19 diagnosis from ct images. Applied Sciences, 11(17), https://doi.org/10.3390/app11178227

33. Daqiu Li, Zhangjie Fu, Jun Xu (2021) Stacked-autoencoder-based model for COVID-19 diagnosis on CT images. Applied Intelligence 51(5):2805-2817. https://doi.org/10.1007/s10489-020-02002-w

34. Layode OF, Rahman Md (2020) A chest X-ray image retrieval system for COVID-19 detection using deep transfer learning and denoising auto encoder. In 2020 International Conference on Computational Science and Computational Intelligence (CSCI), pages 1635-1640, Las Vegas, NV, USA, December. IEEE. https://doi.org/10.1109/CSCI51800.2020.00301

35. Chirag Agarwal, Shahin Khobahi, Dan Schonfeld, Mojtaba Soltanalian (2021) CoroNet: a deep network architecture for enhanced identification of COVID-19 from chest X-ray images. In proceedings of medical imaging 2021: computer-aided diagnosis, volume 11597 of SPIE Medical Imaging 2021, pages 484-490. SPIE, February https://doi.org/10.1117/12.2580738

36. Mansour Romany F, Josè Escorcia-Gutierrez, Margarita Gamarra et al (2021) Unsupervised deep learning based variational autoencoder model for COVID-19 diagnosis and classification. Pattern Recognition Letters 151:267-274. https://doi.org/10.1016/j.patrec.2021.08.018

37. Scarpiniti Michele, Ahrabi Sima Sarv, Baccarelli Enzo, Piazzo Lorenzo, Momenzadeh Alireza (2022) A novel unsupervised approach based on the hidden features of deep denoising autoencoders for covid-19 disease detection. Expert Systems with Applications 192:116366. https://doi.org/10. 1016/j.eswa.2021.116366

38. Hayden Gunraj, Linda Wang, Alexander Wong (2020) COVIDNet-CT: a tailored deep convolutional neural network design for detection of COVID-19 cases from chest CT images. Frontiers in Medicine, 7(608525), December https://doi.org/10.3389/fmed.2020.608525

39. Chest CT-Scan images Dataset, 1st Edition, August 2020. Kaggle Datasets

40. Kwee Thomas C, Kwee Robert M (2020) Chest CT in COVID-19: what the radiologist needs to know. RadioGraphics 40(7):1848-1865. https://doi.org/10.1148/rg.2020200159

41. Diederik P. Kingma and Jimmy Ba (2015) Adam: a method for stochastic optimization. In 3rd International Conference on Learning Representations, ICLR 2015, San Diego, CA, USA, May. arXiv platform

42. Wolfgang Karl Härdle, Marlene Müller, Stefan Sperlich, Axel Werwatz (2004) Nonparametric and Semiparametric Models. Springer-Verlag Berlin Heidelberg, 1st edition, March. https://doi.org/10. 1007/978-3-642-17146-8

43. Fabian Pedregosa, Gaël Varoquaux, Alexandre Gramfort, et al (2011) Scikit-learn: Machine Learning in Python. Journal of Machine Learning Research, 12(85):2825-2830, October. http://jmlr.org/ papers/v12/pedregosa11a.html

44. Habbema J D F, Hermans J, Van den Broeck K (1974) A stepwise discriminant analysis program using density estimation. In Computational Statistics, pages 101-110, Vienna, Austria, INISTCNRS. Pascal and Francis Bibliographic Databases

45. Duin R P W (1976) On the choice of smoothing parameters for Parzen estimators of probability density functions. IEEE Transactions on Computers, C-25(11):1175-1179, November https://doi. org/10.1109/TC.1976.1674577

46. Ethem Alpaydin (2020) Introduction to Machine Learning. MIT Press, 4th edition, March. MIT Press

47. Christian Szegedy, Wei Liu, Yangqing Jia, et al (2015) Going deeper with convolutions. In 2015 IEEE Conference on Computer Vision and Pattern Recognition (CVPR), pages 1-9, Boston, MA, USA, October. IEEE. https://doi.org/10.1109/CVPR.2015.7298594 
48. Alex Krizhevsky, Ilya Sutskever, Geoffrey E Hinton (2012) Imagenet classification with deep convolutional neural networks. In proceedings of the 25th International Conference on Neural Information Processing Systems, NeurIPS'12, pages 1097-1105, Stateline, Nevada, USA, December. Curran Associates Inc. NeurIPS 2012

49. Kaiming He, Xiangyu Zhang, Shaoqing Ren, et al (2016) Deep residual learning for image recognition. In proceedings of the IEEE Conference on Computer Vision and Pattern Recognition, CVPR'16, pages 770-778, Las Vegas, NV, USA, June. IEEE. https://doi.org/10.1109/CVPR.2016. 90

50. Enzo Baccarelli, Michele Scarpiniti, Alireza Momenzadeh et al (2021) Learning-in-the-Fog (LiFo): deep learning meets Fog computing for the minimum-energy distributed early-exit of inference in delay-critical IoT realms. IEEE Access 9:25716-25757. https://doi.org/10.1109/ACCESS.2021. 3058021

51. Enzo Baccarelli, Paola G. Vinueza Naranjo, Mohammad Shojafar, Michele Scarpiniti (2017) Q*: Energy and delay-efficient dynamic queue management in tcp/ip virtualized data centers. Computer Communications, 102:89-106, April. https://doi.org/10.1016/j.comcom.2016.12.010

52. Enzo Baccarelli, Roberto Cusani (1996) Recursive Kalman-type optimal estimation and detection of hidden Markov chains. Signal Processing 51(1):55-64. https://doi.org/10.1016/0165-1684(96) 00030-8

53. Enzo Baccarelli, Mauro Biagi (2003) Optimized power allocation and signal shaping for interference-limited multi-antenna "ad hoc" networks. In M. Conti, S. Giordano, E. Gregori, and S. Olariu, editors, Personal Wireless Communications. PWC 2003, volume 2775 of Lecture Notes in Computer Science, pages 138-152. Springer. https://doi.org/10.1007/978-3-540-39867-7_12

54. Enzo Baccarelli, Mauro Biagi, Cristian Pelizzoni, Nicola Cordeschi (2007) Optimized power-allocation for multiantenna systems impaired by multiple access interference and imperfect channel estimation. IEEE Transactions on Vehicular Technology 56(5):3089-3105. https://doi.org/10.1109/ TVT.2007.900514

Publisher's Note Springer Nature remains neutral with regard to jurisdictional claims in published maps and institutional affiliations. 